\title{
Mycoplasma hyopneumoniae and Mycoplasma hyosynoviae Infection in Cases of Fibrinous Pericarditis in Slaughter Pigs
}

Pericarditis, acute or subacute, is found at post mortem meat inspection of baconers in about 0.02 to $0.04 \%$ of slaughtered pigs in Denmark. The pathological findings are usually restricted to the pericardial sac. The pericardial sac is filled with a fibrinous exudate, which may be blood stained. In some cases massive granula-

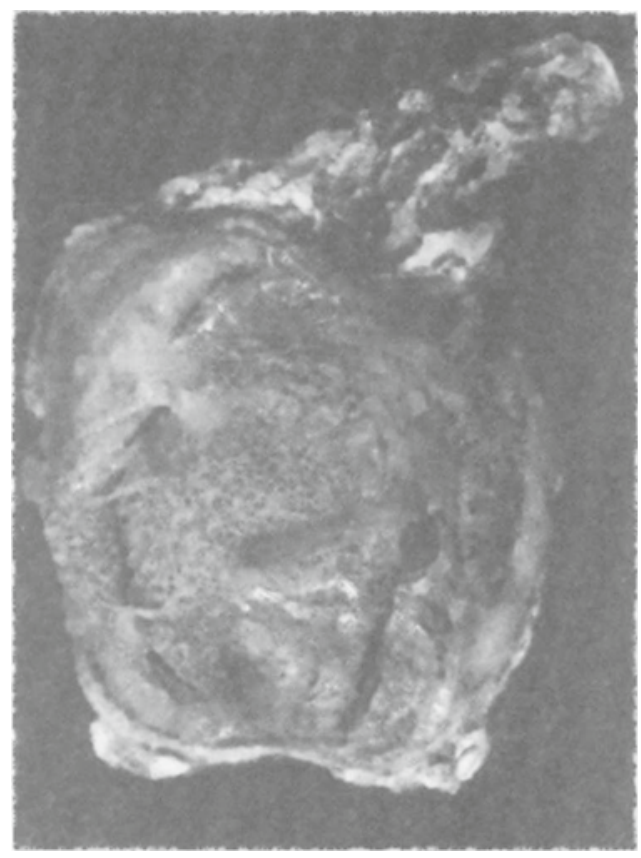

Fig. 1. Heart with chronic fibrinous mycoplasmal pericarditis from a slaughtered pig. Both Mycoplasma hyopneumoniae and M. hyosynoviae were immunohistochemically detected in the exudate. tion tissue formation is seen underlying the fibrinous exudate. Other constantly occurring, but less aggravating lesions, are chronic catarrhal bronchopneumonia and increased volume of serosanguinous synovial fluid in the large joints of the limbs. Lesions usually seen as sequelae to septicaemia have not been observed. The lesions seem to be part of a pathological entity which may be involved in the pathogenesis of fibrinous pericarditis in baconers.

This expression of disease has given rise to speculation about the aetiology, consequences in respect to meat inspection and possible zoonotic aspects. It was therefore decided to examine the disease more closely. This communication describes the preliminary results of the investigation.

From a single minor slaughterhouse, $10100 \mathrm{~kg}$ pigs with fibrinous pericarditis (Fig. 1) were obtained and samples comprising heart with pericardium, lung, elbow joints, spleen, liver and kidneys were transferred to the laboratories involved.

The involvement of pathogenic mycoplasmas was studied histopathologically by applying a triple-layered, indirect immunohistochemical peroxidase-antiperoxidase method (Jensen et al. in preparation) using rabbit antiserum against Mycoplasma hyopneumoniae, Mycoplasma hyosynoviae, and Mycoplasma hyorhinis (Friis \& Jensen 1984).

Cultivation for mycoplasmas was performed on 
Table 1. Immunohistochemical detection of mycoplasma antigens and microbiological culturing from cases of fibrinous pericarditis in $100 \mathrm{~kg}$ slaughter pigs.

\begin{tabular}{rccc}
\hline $\begin{array}{c}\text { Pig } \\
\text { No. }\end{array}$ & $\begin{array}{c}\text { Immunohisto- } \\
\text { chemistry }\end{array}$ & $\begin{array}{c}\text { Mycoplasma } \\
\text { cultivation }\end{array}$ & $\begin{array}{c}\text { Bacteria } \\
\text { cultivation }\end{array}$ \\
\hline 1 & MP,MS & MP & S \\
2 & MP,MS & MP & M \\
3 & MP,MS & MP & M \\
4 & MP,MS & MP & S \\
5 & MP,MS & MP & S \\
6 & MP,MS & MP & S \\
7 & MP,MS & MP & S \\
8 & MP,MS & MP, MS & S \\
9 & MP,MS & MP, MS & M \\
10 & MP,MS & MS & M \\
\hline
\end{tabular}

$\mathrm{MP}=$ Mycoplasma hyopneumoniae

$\mathrm{MS}=$ Mycoplasma hyosynoviae

$\mathrm{S}=$ Sterile

$\mathrm{M}=$ Mixed insignificant flora.

pericardial exudate from all 10 pigs and on pooled synovial fluid from both elbow joints from 7 of the 10 pigs. Bacterial cultivation was performed from all tissue samples. Presence of chlamydia in the pericardial exudate was examined for by use of Chlamydia test kit (Clearview, Unipath).

All 10 pigs were affected with fibrinous pericarditis, chronic catarrhal bronchopneumonia and serosanguinous synovitis in the large joints of the limbs.

Immunohistochemically, both $M$. hyopneumoniae antigen and $M$. hyosynoviae antigen were demonstrated as small mycoplasma-like organisms in the pericardial exudate of all 10 pigs (Table 1) and in 8 of the lungs. M. hyosynoviae antigen only, was demonstrated in the remaining 2 lungs. In the pericardial exudate the mycoplasmas were found adherent to, or intracytoplasmatically in mononuclear cells but also as free solitary organisms. Presence of $M$. hyopneumoniae antigen in the synovial membranes was demonstrated in one joint from 2 pigs. $M$. hyosynoviae antigen was not detected in the synovial membranes.

The presence of mycoplasmas in the pericardial exudate was confirmed by cultivation of $M$. hyopneumoniae from 9 and $M$. hyosynoviae from 3 pigs (Table 1). Thus both mycoplasmas were cultured from 2 pigs. From the 7 pooled synovial fluid samples, $M$. hyopneumoniae was cultured in one case as being the only finding from the joints. The culturing of $M$. hyopneumoniae in this case corresponded to the immunohistochemically demonstration of $\mathrm{M}$. hyopneumoniae antigen.

No growth was obtained on blood agar from pericardial samples taken from 6 of the animals, while a mixed insignificant flora was obtained from the remaining four animals (Table 1). The testing for chlamydia was negative.

While all 10 cases of pericarditis were pathologically uniform and infected with mycoplasmas, the finding of a mixed bacterial flora in the pericardial exudate in 4 of the pigs may be ascribed to contamination. Contamination may be caused by the evisceration procedure of the thorax at slaughtering, during which the pericardium is inevitably punctured.

The immunohistochemical demonstration of concurrent infections with $M$. hyopneumoniae and $M$. hyosynoviae in the pericardial lesions was in one case verified completely by the cultivation. Apparently the method of isolation is less sensitive than the immunohistochemical staining, especially in regard to $M$. hyosynoviae.

To the best of our knowledge these are the first reported cases of both $M$. hyopneumoniae and $M$. hyosynoviae infection of the pericardium, while $M$. hyopneumoniae previously has been isolated from joints with arthritis (Lloyd \& Etheridge 1981). In Denmark, M. hyosynoviae is a common isolate from joints with arthritis (Friis et al. 1992). Although the aetiologic importance of the mycoplasmas in the fibrinous 
pericarditis is not definitely revealed, the results nevertheless indicate the presence of a new mycoplasma related disease syndrome in pigs.

\section{Acknowledgements}

This study was supported by The Federation of Danish Pig Producers and Slaughterhouses.

T.K. Jensen ${ }^{l, *}$, B. Aalbak ${ }^{2}$, J. Buttenschøn ${ }^{3}$, N.F. Friis $^{4}$, J. Kyrval ${ }^{5}$, L. Rønsholt ${ }^{6}$.

${ }^{1}$ Department of Pharmacology and Pathobiology and ${ }^{2}$ Department of Microbiology, Royal Veterinary and Agricultural University, Frederiksberg. ${ }^{3}$ Ministry of Agriculture and Fisheries, Veterinary Services, Vestjyske Slaughterhouses, Allingaabro. ${ }^{4}$ Danish Veterinary Laboratory, Copenhagen. ${ }^{5}$ The Federation of
Danish Pig Producers and Slaughterhouses. ${ }^{6}$ State Veterinary Institute for Virus Research, Lindholm, Denmark. *Present address: Danish Veterinary Laboratory, Copenhagen.

\section{References}

Friis NF, Hansen KK, Schirmer AL, Aabo S: Mycoplasma hyosynoviae in joints with arthritis in abattoir baconers. Acta vet scand. 1992, 33, 205210.

Friis NF, Jensen PT: Serological comparison of type strains of porcine, bovine, and ovine mycoplasmas with atypical colony morphology. Acta vet scand. 1984, 25, 29-35.

Lloyd LC, Etheridge JR: The pathological and serological response induced in pigs by parenteral inoculation of Mycoplasma hyopneumoniae. J. Comp. Path. 1981, 91, 77-83.

(Received January 19, 1995; accepted September 7, 1995).

Reprints may be requested from: T.K. Jensen, Danish Veterinary Laboratory. Bülowsvej 27. DK-1790 Copenhagen V, Denmark. 
